\title{
SEEMP: An Semantic Interoperability Infrastructure for e-Government Services in the Employment Sector
}

\author{
E. Della Valle ${ }^{1}$, D. Cerizza ${ }^{1}$, I. Celino ${ }^{1}$, J. Estublier ${ }^{2}$, G. Vega ${ }^{2}$, M. Kerrigan ${ }^{3}$, \\ J. Ramírez $^{4}$, B. Villazon ${ }^{4}$, P. Guarrera ${ }^{5}$, G. Zhao ${ }^{5}$, and G. Monteleone ${ }^{6}$ \\ ${ }^{1}$ CEFRIEL - Politecnico of Milano, Via Fucini 2, 20133 Milano, Italy \\ 2 Equipe Adele, LSR, Université Joseph Fourier,F-38041 Grenoble Cedex 9, France \\ ${ }^{3}$ DERI, University of Innsbruck, Technikerstraße 21a, 6020 Innsbruck, Austria \\ ${ }^{4}$ Universidad Politecnica de Madrid, 28660 Boadilla del Monte, Madrid, Spain \\ ${ }^{5}$ Le Forem, Boulevard Tirou 104, 6000 Charleroi, Belgium \\ ${ }^{6}$ TXT e-solutions, via Frigia 27, 21126 Milano, Italy
}

\begin{abstract}
This paper presents SEEMP, a marketplace to coordinate and integrate public and private employment services (ESs) around the EU Member States. The need for flexible collaboration in the marketplace gives rise to the issue of interoperability in both data exchange and share of services. SEEMP proposes a mixed approach that relies on the concepts of services and semantics. SEEMP approach combines Software Engineering and Semantic Web methodologies/tools in an infrastructure that allows for a meaningful service-based communication among ESs.
\end{abstract}

\section{Introduction}

SEEMP project1 aims at designing and implementing in a prototype an Interoperability infrastructure for e-government. More specifically, SEEMP is developing an EIF-compliant 1 to allow interoperability among the hundreds of public and private Employment services (ESs) that exist in Europe. The resulting European Employment Marketplace will overcome the national barriers complying, at the same time, with the local policies of each Member States.

Thanks to SEEMP, which promotes increased partnership between labour market actors and the development of closer relations between private and public employment services, job-seekers and employers will have better services that operate at European scale. For instance, the matching process between job offers and CVs across all Europe will become possible increasing, eventually, labour hiring and workforce mobility. In order to fulfil such an ambitious goal several problems must be solved at organizational and technical level.

At an organizational level, the business model of SEEMP has to be catchy for all ESs. The main reason for a ES to buying in is creating added value for its local users (both job seekers and employers) by offering interconnections with

\footnotetext{
${ }^{1}$ SEEMP is funded by EU (IST-4-027347); please visit http://www.seemp.org/.
} 
other ESs. Today it is normal for all users to insert CV and Job Offers in many ESs and collect, laboriously, the results personally. When SEEMP will be in place each ES will be able to collaborate with other ESs. From the perspective of the end user, the add-value is the outreach to other niches of the job market without 'being stretched out". End users could insert the CV and Job Offers once and collect pan-European results. From ESs perspective it will results in increase both the number of users and their faithfulness to each ES, thus an increase in transaction volume.

The need for such flexible collaboration between ESs, gives rise to the issue of interoperability in both data exchange and share of services. The technical approach of SEEMP relies on the concepts of Web Services and semantics. Web Services, exploited in a Software Engineering manner, enable an easier maintenance of the integration. Semantics, encoded in the systems by the means of ontologies and mediators, allows for reconciliation of the hundreds local professional profiles and taxonomies.

SEEMP solution will expose, following the well established Software Engineering approach of Mélusine [2, a single consistent set of abstract services each ES can invoke. Such abstract services will provide a multilateral interoperability solution that delegates the execution of the services to the local ESs (in accordance with the subsidiarity principle) and aggregates the results before sending the response back to the invoker. Moreover, following the innovative Web Service Modeling Ontology [3] approach, we will capture the semantics shared among ESs in a single consistent model. Such model includes a reference ontology in which and from which the local semantics is mapped as well as semantic description of the local Web Services for their automatic use. A set tools will be provided to each ES for modeling its local ontology and for aligning the local ontology with the reference one. As a technical result SEEMP will enable a meaningful service-based communication among ESs.

The paper is structured as follows: Section 2 presents a running example that will be discussed throughout the paper; Section 3 explains the interoperability issue that arises in SEEMP project; Section 4 presents the SEEMP approach to support meaningful service-based communication among ESs; Sections 5 and 6] outline SEEMP solution architecture and components rooting them to the related work in Web Services and Semantic Web community; Section 7 briefly discusses the approach of SEEMP comparing it with already implemented approaches; and finally Section 8 presents future work.

\section{A e-Employment Running Example}

European Member States have introduced major reforms to make the labour market more flexible, transparent and efficient. Such major reforms include decentralization, liberalization of the mediation market (competition between public and private), and quality monitoring of ES staff and services. As an effect ESs understood the need for making available on-line a one-stop shop for the employment. This results in an increased used of ICT and in a boost in differentiating 
and personalizing the services they offer (e.g., Borsa Lavoro Lombardia, Le FOREM 2). For the discussion of this paper we will consider a running example derived by the user requirements of the SEEMP project (for a more detailed description of the socio-economic constrains please refer to [4]):

Job seekers (companies) put their CVs (Job Offers) on a local ES and ask to match them with the Job Offers (CVs) other users put in different ESs through SEEMP.

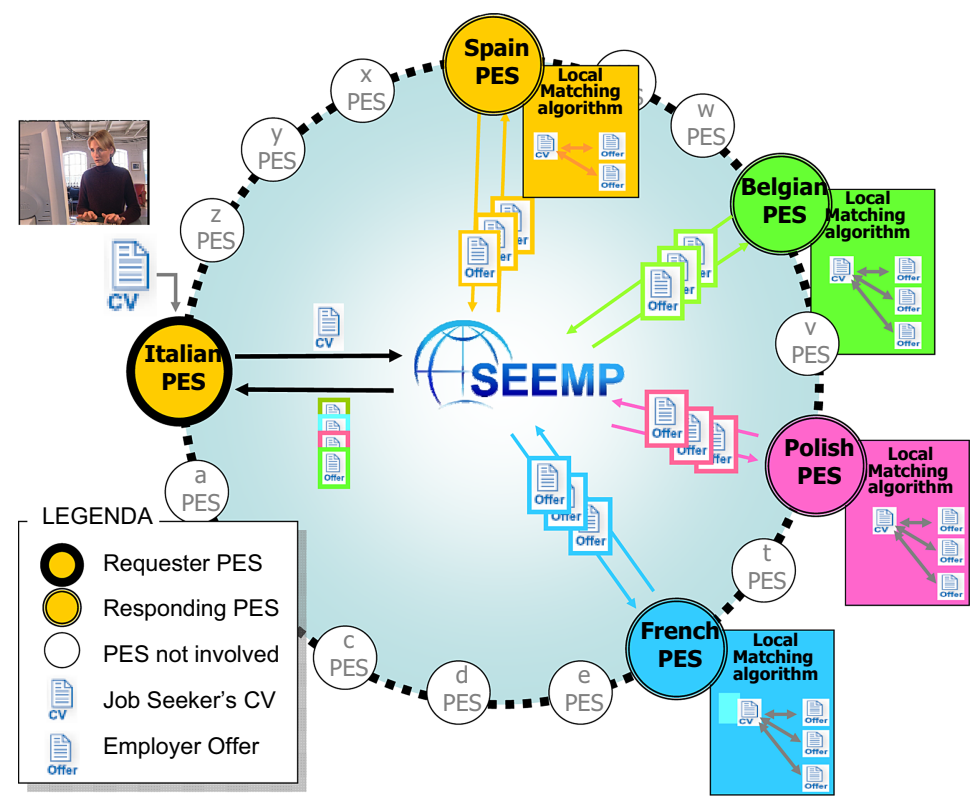

Fig. 1. The running example of distributed matching of CVs and job offers

It may look like a fairly simple example, but to reach its potential EU-wide audience, this e-Employment running example (see figure 1) needs to fulfill a wider set of requirements than the respective local ES services. A local matching service is designed for national/regional requirements only (i.e., central database, single professional taxonomy, single user language, etc.). SEEMP has to be able to send the request, which an end-user submits to the local ES (the Italian ES on left in the figure), to all the other ESs in the marketplace. In order to avoid asking "all" ESs, SEEMP has to select those ESs that most likely will be able to provide an answer and send the request only to them (the four ES on the right in the figure). Moreover, the answers should be merged and ranked homogeneously by SEEMP before they are sent back.

\footnotetext{
$\overline{{ }^{2} \text { Respectively http://www.borsalavorolombardia.net/ and http://www.leforem.be/ }}$
} 


\section{$3 \quad$ Interoperability Issues}

The running example presented in section 2 highlights the need for a system that covers the whole EU and subsumes hundreds of real heterogeneous systems existing in EU countries and regions. It implies by-passing:

- language heterogeneity, e.g., an Italian Java Analyst Programmer may be looking for job offers written in all the different European languages;

- CVs and Job Offers structural heterogeneity, i.e., the use of standards like HR-XML 3 is not wide spread and a multitude of local formats exists;

- CVs and Job Offers content heterogeneity, i.e., European level occupation classifications like ISCO-884 exist, but they do not reflect legitimate differences and perspectives of economic, cultural and political environments;

- heterogeneity of service interface and behavior, i.e., no standard exists for e-employment services thus each ES implemented them differently.

All those are typical interoperability issues. The need for interoperability at European Level among e-Government services has been perceived since 1999 [5] with the adoption of a series of actions and measures for electronic interchange of data between administrations, businesses and citizens (IDABC) [6] .

The main results of IDABC is the European Interoperability Framework (EIF) 11. EIF follows the principle of subsidiarity 5 in addressing the interoperability problem at all levels: organizational, semantic and technical. One crucial aspect, deriving from the principle of subsidiarity, is to keep responsibility decentralized; in other words each partner should be able to keep its own business process almost unchanged 6 and to provide externally point of exchange for its processes. EIF names these points "business interoperability interfaces" (BII).

EIF does not prescribe any solution, but it rather recommends the principles to be considered for any e-Government service to be set up at a pan-European level: accessibility, multilingualism, security, privacy, use of open standards and of open source software and use of multilateral solutions. SEEMP proposes itself as an implementation of EIF in the domain of e-employment.

\section{The SEEMP Approach}

SEEMP relies on the concept of Service. Following the EIF, each ES locally must expose its BII as Web Services. All these Web Services differ but they are fairly similar. SEEMP, as marketplace, models a single consistent set of

\footnotetext{
${ }^{3}$ http://www.hr-xml.org/

${ }^{4}$ http://www.warwick.ac.uk/ier/isco/isco88.html

${ }^{5}$ The principle of subsidiarity recommends not to interfere with the internal workings of administrations and EU Institutions.

${ }^{6}$ Quoting from IDABC: "it is unrealistic to believe that administrations from different Member States will be able to harmonize their business processes because of panEuropean requirements".
} 
Web Service out of those exposed by the ESs. Therefore the services exposed by SEEMP become the standard for the distributed independent service providers.

For instance, for the running example provided in section 2 each ES should expose two Web Services: match an external CV against the job offers stored locally and match an external job offer against the CVs stored locally. Therefore a service that subsume all the local heterogeneous ones can be modeled in SEEMP for each of the two families of similar Web Service. These two abstract service are those that SEEMP, as a marketplace, offers to the ESs.

SEEMP uses Mélusine [2] as tool for modeling those abstract services and orchestrating the process of delegating the execution to the distributed independent service providers.

\section{SEEMP relies on the concept Semantics (both ontologies and media-}

tors. As for the service, each local ES has its own local ontology for describing at a semantic level the Web Services it exposes, and the structure/content of the messages it exchanges. All these ontologies differ but they are fairly similar, because a common knowledge about employment exists as well as the needs for exchange (i.e., you don't exchange on things with no equivalence calculus). So, SEEMP, as marketplace, models a single consistent ontology out of those exposed by the ESs. Therefore the reference ontology of SEEMP becomes the actual standard for the ESs that should provide the mediators for translating from the local ontologies to the reference one and vice versa.

For instance, for the running example each ES should model in a local ontology the structure/content of its $\mathrm{CV} /$ job offers and the way they are exchanged via Web Services. A reference ontology that subsumes all the local heterogeneous ones can be modeled in SEEMP and it becomes the source of shared understanding. Each ES has to provide its mediator for local-reference semantic mapping.

SEEMP adopts WSMO [3] a way to semantically describe Web Service, ontologies and mediators, WSML [7] as concrete syntax for encoding those descriptions and METHONTOLOGY [8] as methodology for developing and maintaining those semantic descriptions.

Minimal shared commitment. In implementing SEEMP approach particular attention is paid in keeping a "win-win" situation among all ESs. The commitment (both at services and semantics level) of each ES should be minimal. ESs care about being able to share while maintaining all the necessary and unnecessary disagreements. It may appear counter-intuitive, but the most suitable set of services and ontologies is the one that enables ESs to "agree while disagreeing". Both the reference set of services and the reference ontology must cover the various aspects of the employment market with an acceptable level of details that leaves leeways of disagreement.

Considering the running example of section 2, the minimal shared commitment the SEEMP consortium agreed upon consists in sharing a subset of the CV named candidacy and a subset of job offer named vacancy. Candidacy (vacancy) enables matching without reveling how to contact the job seeker (employer) that 


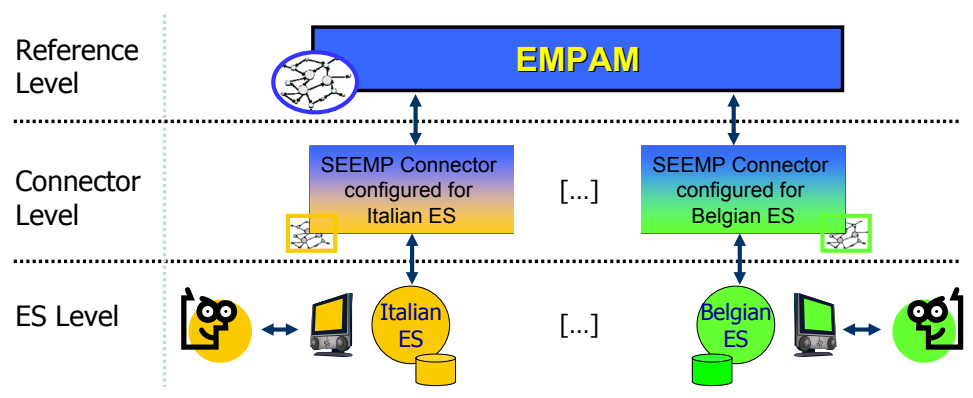

Fig. 2. An overview of the SEEMP solution

is, instead, in the CV (job offer) stored in the ESs and that can be contacted by invoking a service of the ES responsible for the CV (job offer). In this way SEEMP approach also takes into consideration privacy issues of collaborative network, which is a technical issue as well as a business constraint.

\section{The SEEMP Solution Architecture}

SEEMP solution is composed of a reference part (all the dark components in figure 2), which reflects the "minimal shared commitment" both in terms of services and semantics, and by the connectors toward the various local actors (the components in shading colors in figure 2).

\subsection{Structural Overview}

The reference part of SEEMP solution. is made up of the central abstract machine, named EMPAM (Employment Market Place Abstract Machine) and a set of SEEMP services.

The EMPAM is an "abstract machine", in that it does not perform directly any operation, but rather offers abstract services that are made concrete by delegation: when the abstract service is invoked, the EMPAM delegates its execution to the appropriate ES by invoking the correspondent concrete services. It acts as a multilateral solution (as request by EIF), in which all the services connected to the EMPAM are made available to all other ESs, i.e. they ensure a pan-European level of services without interfering with the Business processes of each ES.

The SEEMP services are meant to support EMPAM execution. The running example requires two SEEMP services: discovery and ranking. The discovery service is offered by Glue [9]. The EMPAM invokes Glue Discovery Engine before delegating the execution to the concrete services exposed by the ESs. Glue analyzes the CV sent by the invoking ES and it selects among all ESs those that most likely would be able to return relevant job offers. The ranking service is invoked by the EMPAM after all the concrete services have answered and it 
merges the results providing an homogeneous ranking of the returned job offers. It also delete possible duplicated job offers, which different ESs may have returned.

The SEEMP connectors. enables all communication that occurs between the EMPAM and a given ES. A SEEMP connector will exist for each of the ESs that are connected to the EMPAM and has two main responsibilities:

- Lifting and Lowering: when communicating with the ES any outgoing (or incoming) data which is exchanged by the means of Web Services must be lifted form XML to WSML in terms of the local ontologies of the ES (or lowered back to the XML level from WSML).

- Resolving Heterogeneity: each ES has its own local ontology that represents its view on the employment domain. The SEEMP connector is responsible for resolving these heterogeneity issues by converting all the ontologized content (the content lifted from the XML received from the ES) into content in terms of the reference ontology shared by all partners and vice versa.

\subsection{Functional Overview}

By combining the EMPAM and the connectors SEEMP solution enables a meaningful service-based communication among ESs. Figure 3 illustrates how such meaning communication takes place in running the example of section 2 .

1. the user inserts a CV into the Italian ES and requests job offers,

2. the Italian ES invokes the marketplace matching service passing the CV encoded in the Italian ontology,

3. the SEEMP connector translates the CV from the Italian ontology to the reference one,

4. the discovery SEEMP service analyzes the CV and selects the ESs to be contacted,

5. the EMPAM invokes in parallel the local matching service of selected ESs,

6. the SEEMP connectors of the selected ESs translate the CV from the reference ontology to the local ontology (i.e., the Belgian and the French ESs) and invoke the ES's Web service,

7. the Belgian and the French ES compute the matching locally and returns a set of job offers,

8. each of the connector translates the job offers from each local ontology to the reference one,

9. the ranking SEEMP service merges the responses and ranks the job offers,

10. the job offers are sent back in the reference ontology to the Italian connector that translates them in the Italian ontology,

11. the connector responds to the Italian ES, and

12. finally the Italian ES displays the job offers to the user. 


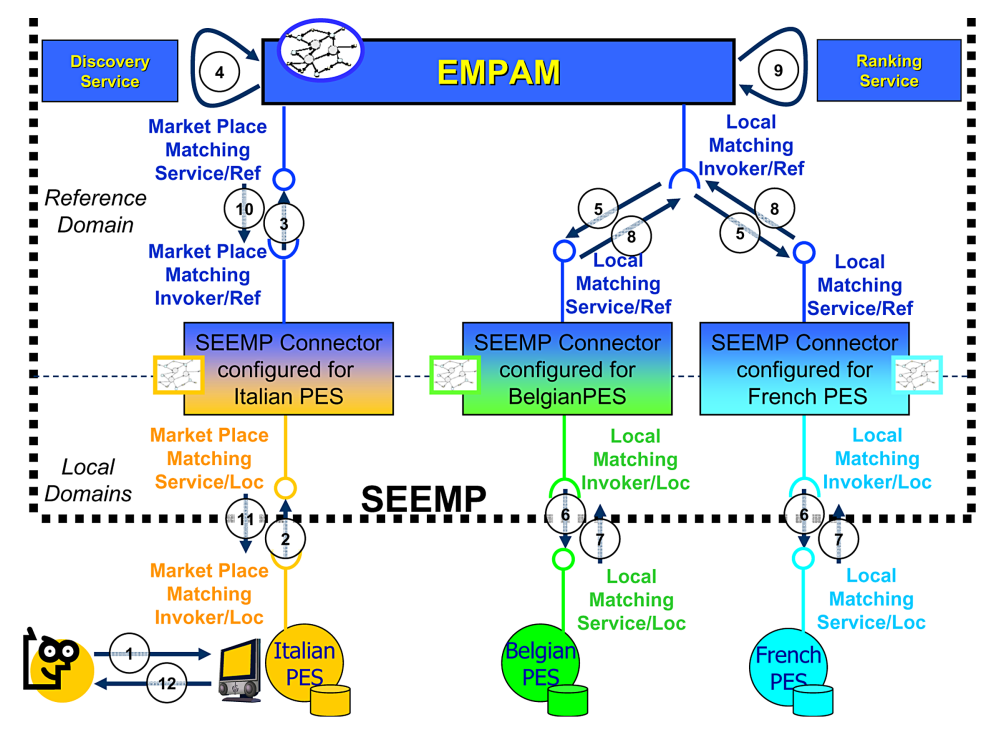

Fig. 3. How SEEMP solution enables meaningful service-based communication

\section{The SEEMP Solution Components}

\subsection{Reference and Local Ontology for e-Employment}

The Reference Ontology is a core component of the system. It acts as a common "language" in the form of a set of controlled vocabularies to describe the details the employment sector. The Reference Ontology has to be rich enough to support the semantic needs of all the ES (Local Ontologies) involved currently and in the future. The Reference Ontology also has to be a scalable, adaptable and maintainable ontology. For all those reason SEEMP follows the METHONTOLOGY [8] approach:

1. specifying competency questions and identified necessities;

2. selecting the standards that cover most of them;

3. semantic enrichment of the chosen standard; and

4. evaluating the Ontology content.

In order to build the Reference Ontology, the standards identified are: ISO 4217 for currencies, the 12 levels of driving license recognized by the European legislation, NACE Rev. 1.1 for economic activities, ISCO-88 (COM) and ONET taxonomy of occupations, FOET and ISCED97 for education, ISO 3166 country codes, LE FOREM classifications of contract types and work rule types, ISO 6392 for languages, and EDS classification for skills.

Local Ontologies. Based on the proposed SEEMP architecture, the possible options for building the local ontologies in SEEMP ranges between to extreme 
options: building local ontologies taking as a seed the reference ontology and building local ontologies as a reverse engineering process from ES schema sources.

In building local ontologies taken as a seed the reference ontology, the concepts in the local ontology are extension in depth of the concepts already present in the reference ontology. By extension we mean including application dependent concepts that appear in each ES schema source.

The exchange of job offers and CV (once ontologized), required by the running example of section 2 is easy because all local ontologies extends the same reference vocabulary. On the contrary mappings complexity are on local ontology and schema mappings (cf. section 6.3 for more details).

Building local ontologies as a reverse engineering process from ES schema sources, is the easiest way for ontologizing ESs. Each concept in the local ontology is the semantic expression of a relevant concept in the ES. In this way ESs becomes ontology-based applications that are more efficient because mappings between local ontologies and schema sources should not be complex, but complex mappings appear between the local and reference ontology. Therefore data exchange will require more time, in comparison to the previous option, due to the execution of two complex mappings.

The SEEMP way is keeping close to the first option in the beginning, when few ESs are in the marketplace and the union of ES semantics is the most straight forward solution. Then while more and more ESs would be added to the marketplace, the solution would move toward the second option. The equilibrium between the two extreme solutions is related to the need for a "minimal shared commitment" explained in section 4.

\subsection{An Employment Market Place Abstract Machine}

The EMPAM machine is implemented as a Mélusine application, which means it is structured following the Mélusine approach in three layers (cf. Figure 4).

Layer 1: The abstract machine. The higher EMPAM machine layer is a java abstrct program where abstract classes represent the concepts present in SEEMP Employment Marketplace Platform (EMP). EMP acts as a ES covering completely EU, i.e. it acts as if all the $\mathrm{CV}$ and vacancies were present in its repositories. However the EMP is abstract since it does not have any information locally, but delegates to real ESs the duty to process part of the job. The EMP program defines functions like matching $\mathrm{CV}$ and job offers that are, indeed, not implemented at all, or only sketching what they are supposed to do.

Layer 2: The adapters. The second layer duty is to do in such a way that empty or dummy methods, found in the abstract layer, really perform what they are supposed to perform. To that end this layer is itself structured in three layers:

- The injection machine, whose duty is to capture those methods that need to be implemented, and to transfer the call to the following layer.

- The mediation and orchestration layer which is in charge of transforming a single abstract method call into a potentially complex orchestration of real lower level services that together will perform the required function. 


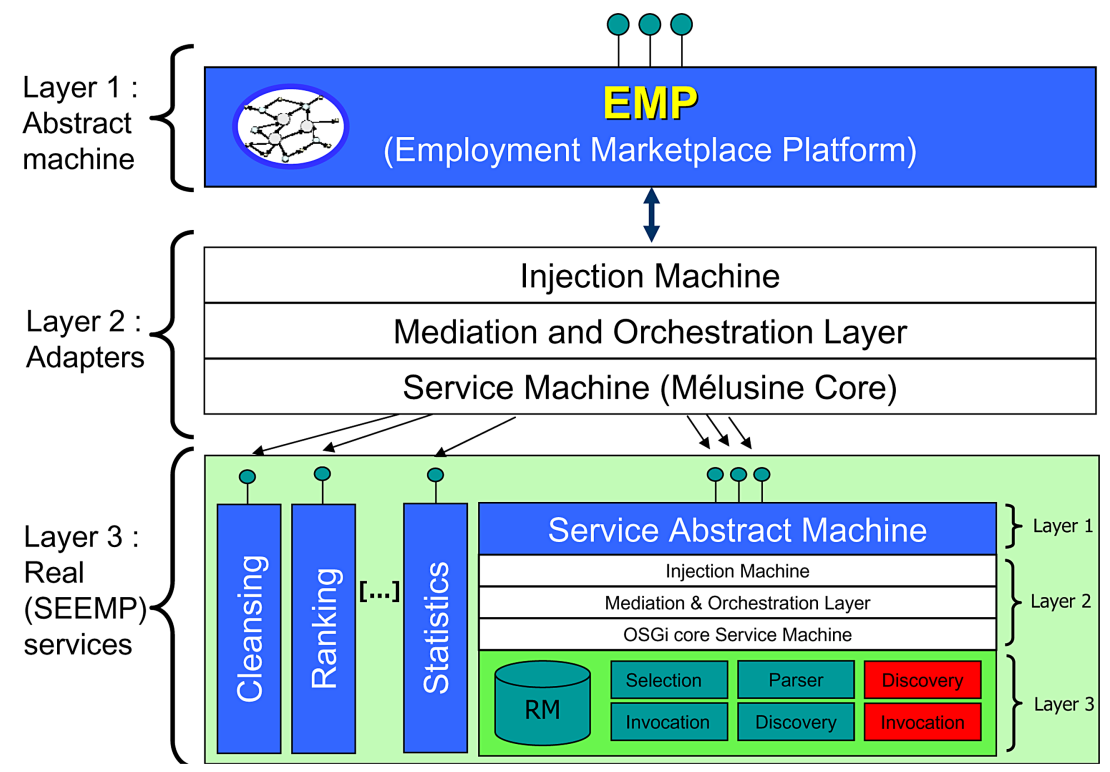

Fig. 4. The levels that made up the EMPAM as a Mélusine application

- The Service machine, whose duty is to transparently find and load the required SEEMP service and to call them. In SEEMP, this service machine is the core Mélusine service machine (an implementation of OSG77).

Layer 3: The SEEMP services, which are OSGi services, and are called accordingly by Mélusine. This solution ensures optimal performance to the EMPAM, while allowing large facilities to future extensions (new SEEMP service) and even dynamic changes (dynamic loading/unloading of services).

Two classes of services have been identified:

- those dedicated to calling a Web Service exposed by a ES through the Service Abstract Machine (SAM). Most of the issues raised by EMP are related to discovering, selecting, parsing, and finally invoking a remote service; more exactly the SEEMP connector of each ES. SAM is itself a Mélusine application and therefore contains itself an abstract layer in which are defined the fundamental concepts and functions of a service machine. This layer is captured and delegated to an orchestration layer that calls local services which, in the scope of SEEMP, are WSMX components [10] wrapped as OSGi services.

- the other service; currently these services, in SEEMP, include the cleansing, ranking and statistic functions, and will include, in the future, the implementation of the specific functions and repositories of the EMPAM machine i.e. those functions and information not available in the ESs. Functions and information available in ESs are available calling the SAM service.

\footnotetext{
7 http://www.osgi.org/
} 


\subsection{SEEMP Connectors}

The SEEMP connectors behave as the mechanism through which all communication occurs between the EMPAM and a given ES. A SEEMP connector will exist for each ES that is connected to the EMPAM and has two main responsibilities: Lifting/Lowering and Resolving Heterogeneity.

Lifting and Lowering: The ESs involved in the SEEMP marketplace only deal in terms of structured XML content and do not deal in terms of ontologies. Within the SEEMP marketplace it is important that all content is ontologized so that it can be reasoned about, thus the SEEMP connector must lift all messages received from a given ES to the ontology level. This is done by converting the XML content received to WSML in terms of the local ontologies of the ES. When communicating with the ES any outgoing data must be lowered back to the XML level so that the ES can understand the content.

Since WSMO elements can be serialized in a RDF format, this task could be done by converting XML content to RDF first, and then converting RDF to WSML. In SEEMP this task is achieved by the means of an extension to $\mathrm{R}_{2} \mathrm{O}$ language [1], which enables to describe mappings between XML schemas and ontologies, and to its related processor ODEMapster [12].

Resolving Heterogeneity: Each ES talks in its own language, essentially having its own local ontology that represents its view on the employment domain. The SEEMP connector is responsible for resolving these heterogeneity issues by converting all the ontologized content (the content lifted from the XML received from the ES) into content in terms of the SEEMP reference ontology. By doing this all the ESs in the marketplace talk in the same language, and thus heterogeneity issues are resolved. Similar to the lowering back to XML, when communicating with a given ES the SEEMP connector is also responsible for converting back from the reference ontology to the local ontology of the given ES.

As described in section 6.1 the reference ontology represents the bridge, or common vocabulary, that the ESs will communicate through. Rather than managing mappings between every possible ontology pair, which essentially becomes unmanageable once a number of ESs have joined the marketplace, each ES need only maintain mappings to and from the reference ontology. These mappings represent a set of instructions (or rules) on how to convert an instance from the local ontology to an instance of the reference ontology (and vice versa).

Technologically this is achieved using the WSMX Data Mediation [13. This work is made up of two components, the first being the design time component, within which the ES will describe the mappings between their local ontology and the reference ontology, and the second being the run time component, which is responsible for executing the mappings at run time to transform the messages between ontologies.

A reusable SEEMP connector is built by bringing together the functionality described above. The architecture of the SEEMP Connector outlined in the 


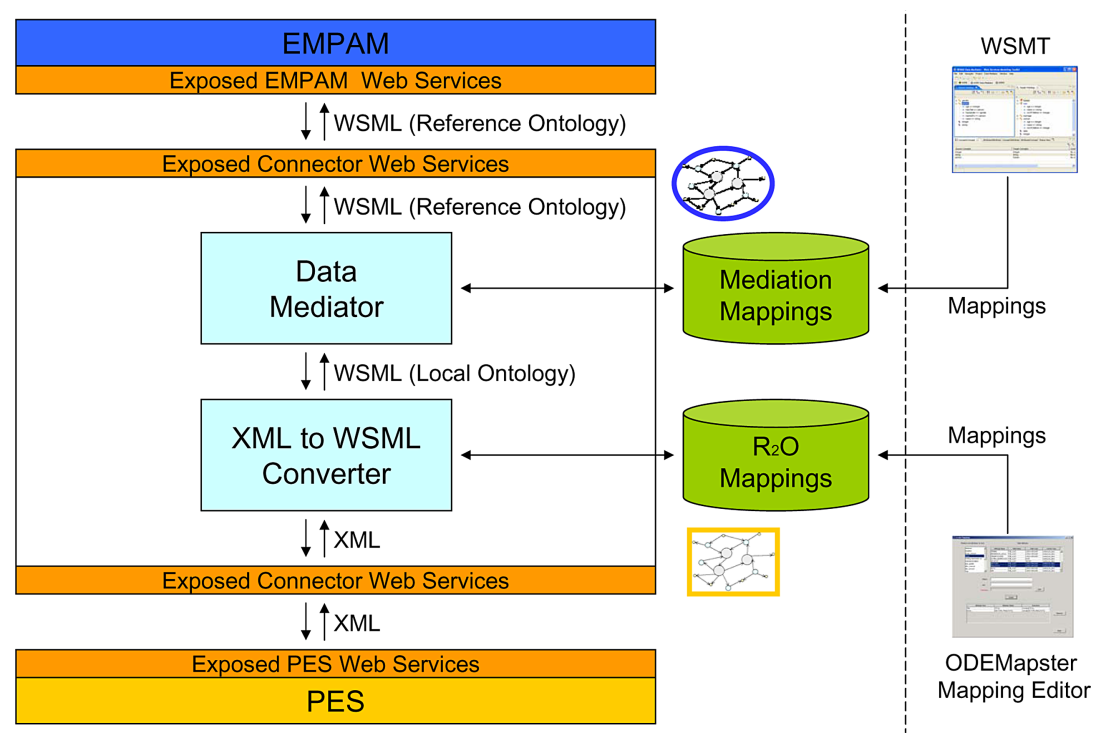

Fig. 5. The SEEMP Connector Architecture

figure 5 shows the ES communicating with the connector using XML via the exposed web services. This XML is then lifted to the local ontology using the $\mathrm{R}_{2} \mathrm{O}$ mappings stored in the repository and furthermore converted to the reference ontology using the data mediation mappings. Ultimately the EMPAM is invoked using messages in the reference ontology via its exposed web services. Communication also occurs in the opposite direction.

Each of the ESs joining the marketplace will require its own SEEMP connector, however the only difference between any two connectors is the code for executing the ESs exposed Web Services as each ES will expose services in a different way. The need for individual SEEMP connectors could be removed through the use of WSMO Choreography 3. to describe the interfaces of the ES services and the integration of the WSMX choreography engine [14 and invoker into the SEEMP Connector, however this was not considered for the first prototype of the SEEMP solution.

\section{Comparing SEEMP with Other Approaches}

In order to draw a comparison between SEEMP and other approaches we selected two case studies: private employment networks (e.g. Adecco) and hierarchical network (e.g. Borsa Lavoro Lombardia, EURES). Moreover we consider the differences both from the point of view of CEO (the decision makers) and CTO (the IT experts).

Compared to other approaches SEEMP solution offers CEO a way to enforce subsidiarity principle, therefore valuing each ES contribution in the marketplace. 
In private networks the subsidiarity principle is not applicable, while in hierarchical networks most of the nodes are passive actors. Moreover the marketplace creates added value by increasing the number of interconnections, hence resulting in more faithful users (more $\mathrm{JO} / \mathrm{CV}$ accessible using the user language) and in more transactions. Many job offers that today could be found only at the cost of inserting the CV multiple times and merging manually the results of different ESs, becomes available through the interface of each ES.

For CTO SEEMP solution enable an easier maintenance of the integration with other ESs and minor integration costs. It was proved that Web Services used in a Service Oriented Architecture easies integration and maintenance. Moreover semantics make mapping different terminology easier because tools (such as WSMT [15]) can analyzed local and reference ontology (e.g., by comparing sub-structures and by searching for synonymies) and can guide the IT Administrator in drawing the mappings. Thank to this support, the mapping definition process requires less time or, eventually, it provides more precise mappings in the same amount of time. That support comes out with a minor integration costs.

In order to achieve this benefit CEO has to develop "partnership", i.e., the ability to collaborate with other peers, ES or staffing industries. The partnerships are different in the two case studies. In private network everything is agreed in advance. In hierarchical network partnership are necessary, but no peer to peer decision taking is possible. Decisions are institutionally imposed top-down. Moreover SEEMP supports CTO with comprehensive set of tools and methodologies for service and semantic interoperability.

Concerning services CTO has to expose ES APIs as Local Web Services and has to provide support for invoking EMPAM services. However, they don't have to understand interfaces and behavior of other ES (as in hierarchical solutions) because the connector presents the market place as if the ES was invoking its own services.

Concerning semantics, CTO has to model data structure and content and has to defining mappings with the reference ontology, but, as discussed above, this is easier and more precise than it is nowadays without ontologies and mediators.

What has to be built, and SEEMP alone won't be able to do, is a comprehensive reference ontology and abstract service machine that encompasses several employment domains. Developing and maintenance this reference part of SEEMP more then a ICT problem; it is a matter of reaching agreement at organizational level. As already discussed in section 4 the goal of SEEMP is reaching a "minimal shared commitment" in which ESs agree on high-level aspects, allowing for collaboration among them, while disagreeing on minor details that differentiate one ES from the others.

\section{Conclusions and Future Work}

This paper presented the SEEMP approach in supporting meaningful servicebased communication among public and private employment services. The following results have been shown: 
- services and semantics are the key concepts for abstracting from the hundreds of heterogeneous systems already in place that are evolving separately. They provide a straight forward way to implement the subsidiarity principle.

- the combination of an abstract service machine with a reference ontology is a technically sound approach to multi-laterality for marketplace implementation. Each actor in the marketplace has to care only about integrating with the marketplace. The marketplace will offer services to support the interaction with the other actors.

- a mix of Software Engineering and Semantic approach is required to achieve flexibility. The two approaches nicely complement each other. By means of "conventional" software engineering design SEEMP build an abstract machine that can run on "conventional" technology and at the same time embeds semantics both in the form of ontology/mediator and in the form of semantic-aware components (i.e. ODE mapster, WSMX data mediation, Glue Discovery Engine).

Currently SEEMP consortium is running a pilot that shows the integration of EURES and Borsa Lavoro Lombardia. This integration has allowed for so far testing the functional aspects of SEEMP approach. The next step is integrating Le FOREM ES as a validation case. We expect, the reference ontology and the abstract machine to be so well designed that Le FOREM introduction would have no impact on them.

Future work includes extending the number of abstract services included in the EMPAM and the respective concepts in the reference and local ontologies. For instance, one essential service of SEEMP should be the possibility to have regularly (monthly, weekly, daily, ) a set of key indicators regarding labour market in all participant regions (job seekers, job offers, training, etc.), in a common and comparable language, both in terms of methods (definitions, calculation of indicators, etc.) and in terms of technical requirements.

\section{Acknowledgments}

The research has been supported by the SEEMP (IST-4-027347-STREP) EU funded project. We thank all the colleagues for the fruitful discussion we have and in particular Flavio De Paoli, Mirko Cesarini and Maria Grazia Fugini.

\section{References}

1. European Communities: European interoperability framework for pan-european egovernment services. Technical report, Office for Official Publications of the European Communities (2004)

2. Estublier, J., Vega, G.: Reuse and variability in large software applications. In: ESEC/SIGSOFT FSE. (2005) 316-325

3. Fensel, D., Lausen, H., Polleres, A., de Bruijn, J., Stollberg, M., Roman, D., Domingue, J.: Enabling Semantic Web Services - The Web Service Modeling Ontology. Springer (2006) 
4. Della Valle, E., Cerizza, D., Celino, I., Estublier, J., Vega, G., Kerrigan, M., Ramírez J., Villazon, B., Guarrera, P., Zhao, G., Monteleone, G.: SEEMP: Meaningful service-based collaboration among labour market actors. In: proceedings of BIS 2007, LNCS 4439, Poznan, Poland, Springer-Verlag (2007)

5. 1720/1999/EC: Decision of the European Parliament and of the Council of 12 July $1999(1999)$

6. 2004/387/EC: Decision of the European Parliament and of the Council on Interoperable Delivery of pan-European Services to Public Administrations, 2004 (2004)

7. de Bruijn, J., Lausen, H., Polleres, A., Fensel, D.: The web service modeling language: An overview. In: Proceedings of the 3rd European Semantic Web Conference (ESWC2006), Budva, Montenegro, Springer-Verlag (2006)

8. Gómez-Pérez, A., Fernández-López, M., Corcho, O.: Ontological Engineering. Springer Verlag (2003)

9. Della Valle, E., Cerizza, D.: The mediators centric approach to automatic web service discovery of glue. In: MEDIATE2005. Volume 168 of CEUR Workshop Proceedings., CEUR-WS.org (2005) 35-50

10. Haller, A., Cimpian, E., Mocan, A., Oren, E., Bussler, C.: WSMX - A Semantic Service-Oriented Architecture. In: ICWS. (2005) 321-328

11. Barrasa, J., Corcho, O., Gómez-Pérez, A.: R2O, an extensible and semantically based database-toontology mapping language. In: Second International Workshop on Semantic Web and Databases. (2004)

12. Rodriguez, J.B., Gómez-Pérez, A.: Upgrading relational legacy data to the semantic web. In: WWW '06: Proceedings of the 15th international conference on World Wide Web, New York, NY, USA, ACM Press (2006) 1069-1070

13. Mocan, A., Cimpian, E., Kerrigan, M.: Formal model for ontology mapping creation. In: International Semantic Web Conference. (2006) 459-472

14. Cimpian, E., Mocan, A.: WSMX Process Mediation Based on Choreographies. In: Business Process Management Workshops. (2005) 130-143

15. Mocan, A., Cimpian, E.: Mappings creation using a view based approach. In: MEDIATE2005. Volume 168 of CEUR Workshop Proceedings., CEUR-WS.org (2005) 97-112 